\title{
Luxation post-traumatique de la hanche chez un enfant
}

\section{Children post-traumatic dislocation of a hip}

\section{Chinellato}

Reçu le 27 septembre 2014; accepté le 10 novembre 2014

(C) SFMU et Lavoisier SAS 2014

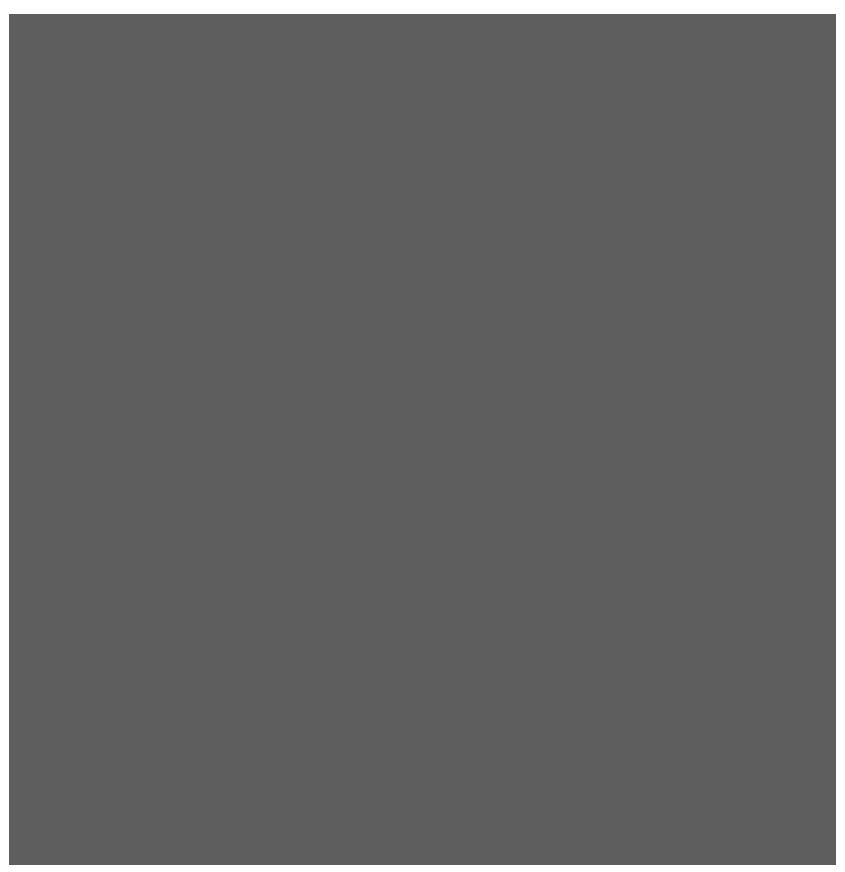

Un garçon de deux ans est retrouvé coincé sous un portail dont les gonds ont cédé. À la prise en charge, il présente une abduction, une flexion et une rotation externe de la hanche droite, douloureuse et irréductible. À son arrivée aux urgences, le bilan radiographique (Fig. 1) confirme la luxation antérieure de la hanche droite, pure c'est-à-dire sans lésion osseuse ou vasculonerveuse associée. Le traitement a consisté en une réduction sous anesthésie générale deux heures après l'accident puis en la pose d'un plâtre pelvipédieux pendant 30 jours. Le contrôle radiologique et orthopédique à l'ablation de l'immobilisation n'a pas montré de complication secondaire et l'examen clinique à trois mois est strictement normal.

La luxation traumatique de la hanche chez l'enfant est une pathologie rare, représentant seulement $5 \%$ des luxations de hanche, tout âge confondu [1]. Elles diffèrent essentiellement de celles de l'adulte par leur rareté et leur meilleur pronostic [2].

\section{Références}

1. Figueras Coll G, Torrededia Del Rio L, Garcia Nuño L, et al (2010) Traumatic hip dislocation in childhood. Hip Int 20:524-8

2. Vialle R, Odent T, Pannier S, et al (2005) Traumatic hip dislocation in childhood. J Pediatr Orthop 25:138-44 